\title{
Performance of a lateral flow test for the detection of leprosy patients in India
}

Leprosy is the result of chronic infection of skin and nerves with Mycobacterium leprae. Many M. leprae-specific as well as Mycobacterium genus-specific antibodies are found in the sera of leprosy patients. Although such antibodies are not known to be protective, their presence in the sera of leprosy patients may be employed for detection of $M$. leprae infection. Thus far, serological tests using phenolic glycolipid-I (PGL-I), a unique component of $M$. leprae (Hunter \& Brennan, 1981), have been most commonly used for detection of $M$. leprae infection (Oskam et al., 2003). The organism is known to induce,

predominantly, an IgM type of antibody response (Cho et al., 1983). Of the various potential serological assays, ELISA-based tests have been most commonly assessed for their value in the diagnosis of leprosy, in monitoring the effectiveness of chemotherapy, in detecting the emergence of relapse, in identifying patients with a high risk of reactions during therapy and in monitoring changes in the magnitude of $M$. leprae infection in a population (Oskam et al., 2003). Although ELISA is a versatile tool, it has some drawbacks such as its requirements for trained personnel, expensive equipment and consumables that need to be stored in cool conditions. All these are discouraging for the settings where leprosy is most prevalent. Moreover, it takes nearly 1 day before results are available. Hence a more convenient, rapid, robust and easy-to-use test is desirable.

Over the years, some simple-to-perform diagnostic assays (Izumi et al., 1990; Buhrer-Sekula et al., 1998) have been reported as an alternative to anti-PGL-I antibody detecting ELISA. In the recent past, the use of the $M$. leprae lateral flow test (ML Flow test) has been described (for patients from Brazil, Indonesia and the Philippines), which detects antibodies to PGL-I within 10 min (Buhrer-Sekula et al., 2003). The sensitivity of the test has been described to be $97.4 \%$ for multibacillary (MB) and $40 \%$ for paucibacillary (PB) leprosy patients. Recently, Buhrer-Sekula et al. (2007) published their findings (with patients from Brazil, Nepal and Nigeria) on the use of the ML Flow test as an additional serological tool for classification of new leprosy patients. The authors report that the ML Flow test could be used to strengthen classification, reduce the risk of under-treatment and minimize the need for slit skin smears. Here, we have extended the foregoing studies by evaluating the performance of the ML Flow test as a tool for detection of $M$. leprae infection in leprosy patients from India.

Newly diagnosed leprosy patients attending the clinic of the National JALMA Institute for Leprosy and other Mycobacterial Diseases, Agra, India, were enrolled for the study. Patients were diagnosed by the clinical criteria suggested by WHO (1998). A person having any one or more of the features hypopigmented or reddish skin lesion(s) with definite loss of sensation, involvement of the peripheral nerves, as demonstrated by definite thickening with loss of sensation, and skin smear positive for acid-fast bacilli (AFB) was considered a leprosy patient. The study included 147 leprosy patients. Patients whose routine microscopic examination of slit-skin smear was positive for AFB were grouped (WHO, 1988) as MB while those with absence of AFB were grouped as $\mathrm{PB}$. Blood samples from 10 tuberculosis patients, 27 patients with non-leprosy skin diseases and 25 healthy individuals served as negative controls. Informed consent for skin smear and blood samples was obtained from all study subjects.

The ML Flow test kit was acquired from KIT (Biomedical Research). The principle and details of the test have been reported elsewhere (Buhrer-Sekula et al., 2003). Briefly, testing was performed according to the instructions outlined in the product insert. When testing, $5 \mu \mathrm{l}$ whole blood is added to the specimen well of the assay device. Then $130 \mu \mathrm{l}$ reagent-releasing buffer is added to the sample well to release the colloidal gold-labelled goat anti-human IgM antibodies. The sample flows towards the reagent pad, where a colloidal gold-labelled anti-human IgM antibody also joins the moving buffer. Eventually, the moving buffer along with the antibodies encounters the antigen line as well as that of the IgM antibody (deposited as a positive control) in the form of separate lines on the nitrocellulose paper strip. The test results are considered valid only when the control line is clearly visible. The test is scored positive when a distinct staining of the test line is observed within $10 \mathrm{~min}$.

On classifying 147 leprosy patients using skin smear examination, 25 patients were found to be positive for AFB and classified as $\mathrm{MB}$, and 122 patients were observed to be negative and classified as $\mathrm{PB}$. Considering serological results (Table 1), $92.0 \%(23 / 25)$ of the MB patients were serologically positive by the ML Flow test, whereas only $32.0 \%(39 / 122)$ of $\mathrm{PB}$ patients were found to be positive. However, all of the controls (including 10 tuberculosis patients, 27 other skin disease patients and 25 healthy individuals) were negative by the assay. Further, in comparison to the skin smear test for AFB, the sensitivity of the serological assay was better (chi value 22.4; $P<0.001$ ). In a study conducted in a geographically different population, Buhrer-Sekula et al. (2003) reported a slightly higher sensitivity for smear-positive MB patients (97.4\%) as well as for smear-negative $\mathrm{PB}$ patients (40.0\%) with high $(90.0 \%)$ specificity for detection of leprosy. Nevertheless, the findings regarding the performance of the ML Flow test with Indian patients (in our study) were statistically highly similar (chi value $0.36 ; P>0.5)$ to those of Buhrer-Sekula et al. (2003).

Generally, identification of AFB in skin smears is used to confirm the clinical diagnosis of leprosy. Further, detection of AFB in slit skin smears is known to show 
Table 1. Performance of the ML Flow test with various groups

\begin{tabular}{|lrccc|}
\hline \multirow{2}{*}{ Group } & $\boldsymbol{n}$ & \multicolumn{2}{c|}{ ML Flow test result } & \multirow{2}{*}{ Positivity (\%) } \\
\cline { 3 - 4 } & & Positive (MB) & Negative (PB) & \\
\hline Smear-positive (MB) & 25 & 23 & 2 & 92.0 \\
Smear-negative (PB) & 122 & 39 & 83 & 32.0 \\
Tuberculosis & 10 & 0 & 10 & 0.0 \\
Other skin diseases & 27 & 0 & 27 & 0.0 \\
Healthy & 25 & 0 & 25 & 0.0 \\
\hline
\end{tabular}

high specificity but low sensitivity. Hence almost always PB patients are not detected by this method. Some reports show that the smear test fails, due to hidden bacteria, to represent the true bacterial load in a host (Ridley, 1955; Ponnighaus et al., 1997; Srinivas et al., 2002). Since the presence of $M$. leprae in the host generates anti-M. leprae antibodies, blood circulating through the body tissues would carry such antibodies in it from the various irrigated tissues. Hence, circulating anti-M. leprae antibodies have been shown to reflect the presence of $M$. leprae in the host (Douglas et al., 1988; Klatser et al., 1989; Roche et al., 1990). Therefore, detection of anti-PGL-I antibodies is considered to be worthwhile as an alternative to skin smear for the diagnosis of leprosy patients. Since antibodies to PGL-I are known to reflect the bacterial load in the host, the seropositivity in smear-negative cases could be due to antibodies generated by such hidden bacteria in the host. Thus serological testing for PGL-I antibodies may be of some limited value $(32.0 \%$ as observed in the present study and $40.0 \%$ as reported by Buhrer-Sekula et al., 2003) in the diagnosis of patients who present with signs of leprosy but with absence of AFB in smears. Otherwise, patients having hidden bacteria inside their body might be incorrectly considered as non-leprosy. Generally, in early MB leprosy, skin lesions are vague and difficult to see or are invisible, thereby creating a problem in the diagnosis of leprosy. Since the sensitivity of the described assay for the detection of $\mathrm{MB}$ leprosy is very high $(92.0 \%)$, the test could be a useful tool to confirm such doubtful MB leprosy cases (who need longer treatment) as well. Otherwise, undetected cases may remain untreated and thereby could cause deformity in the patient and transmission in the community. Thus, our findings with patients from India are very much in line with the earlier reports of Buhrer-Sekula et al. (2003, 2007).

Serological methods for diagnosis are considered attractive because of their easy implementation under the conditions commonly encountered in developing countries. Since the identification of PGL-I as a $M$. leprae-specific antigen, considerable progress towards development of simple assays such as ELISA, the gelatin particle agglutination test and the ML Dipstick assay (Cho et al., 1983; Izumi et al., 1990; Buhrer-Sekula et al., 1998, 2003) has occurred.

Considering our past experiences (Parkash et al., 1995; Parkash \& Girdhar, 2001), we have found, in the present study, the ML Flow test to be comparatively simpler and more rapid to perform than the ELISA and agglutination tests. Also, in the recent past, the test has been described to be more userfriendly than the Dipstick assay (BuhrerSekula et al., 2003). Over and above, in comparison with ELISA and the gelatin particle agglutination test, the ML Flow test can be conducted using whole blood, thus avoiding the time delay due to serum separation. Additionally, with this test it is very easy to read the results visually, and it does not require any special training or equipment or electricity. In conclusion, the assay is worth employing in leprosy-related investigations to facilitate disease management in remote areas where laboratory facilities are not readily available.

\section{Acknowledgements}

Thanks are due to the Council of Scientific and Industrial Research and the Indian Council of Medical Research for financial support for the study.

\section{Om Parkash, Avnish Kumar, Richa Pandey, Astha Nigam and Bhawneshwar Kumar Girdhar}

National JALMA Institute for Leprosy and Other Mycobacterial Diseases, Taj Ganj, Agra-1, India

Correspondence: Om Parkash (om1234@gmail.com)

Buhrer-Sekula, S., Cunha, M. G., Ferreira, W. A. \& Klatser, P. R. (1998). The use of whole blood in a dipstick assay for detection of antibodies to Mycobacterium leprae: a field evaluation. FEMS Immunol Med Microbiol 21, 197-201.

Buhrer-Sekula, S., Smits, H. L., Gussenhoven, G. C., van Leeuwen, J., Amador, S., Fujiwara, T., Klatser, P. R. \& Oskam, L. (2003). Simple and fast lateral flow test for classification of leprosy patients and identification of contacts with high risk of developing leprosy. J Clin Microbiol 41, 1991-1995.

Buhrer-Sekula, S., Visschedijk, J., Grossi, M. A. F., Dhakal, K. P., Namadi, A. U., Klatser, P. R. \& Oskam, L. (2007). The ML flow test as a point of care test for leprosy control programmes: potential effects on classification of leprosy patients. Lepr Rev 78, 70-79.

Cho, S. N., Yanagihira, D., Hunter, S. W., Gelber, R. H. \& Brennan, P. J. (1983). Serological specificity of phenolic glycolipid I from Mycobacterium leprae and use in serodiagnosis of leprosy. Infect Immun 41, 1077-1083.

Douglas, J. T., Steven, L. M., Fajardo, T., Cellona, R. V., Madarang, M. G., Abalos, R. M. \& Steenbergen, G. J. (1988). The effects of chemotherapy on antibody levels in lepromatous patients. Lepr Rev 59, 127-135.

Hunter, S. W. \& Brennan, P. J. (1981). A novel phenolic glycolipid from $M$. leprae possibly involved in immunogenicity and pathogenicity. J Bacteriol 147, 728-735.

Izumi, S., Fujiwara, T., Ikeda, M., Nishimura, Y., Sugiyama, K. \& Kawatsu, K. (1990). Novel gelatin particle agglutination test for serodiagnosis of leprosy in the field. J Clin Microbiol 28, 525-529.

Klatser, P. R., de Wit, M. Y. L., Fajardo, T. T., Cellona, R. V., Abalos, R. M., de la Cruz, E. C., Madarang, M. G., Hirsch, D. S. \& Douglas, J. T. (1989). Evaluation of Mycobacterium leprae antigens in the monitoring of a dapsone-based chemotherapy of previously untreated lepromatous patients in Cebu, Philippines. Lepr Rev 60, 178-186.

Oskam, L., Slim, E. \& Buhrer-Sekula, S. (2003). Serology: recent developments, strengths, limitations and prospects: a state of the art overview. Lepr Rev 74, 196-205.

Parkash, O. \& Girdhar, B. K. (2001). A comparative and combinatorial study on two serological assays for detection of Mycobacterium leprae infection. Acta Leprol 12, 63-69. 
Parkash, O., Chaturvedi, V., Girdhar, B. K. \& Sengupta, U. (1995). A study on performance of two serological assays for diagnosis of leprosy patients. Lepr Rev 66, 26-30.

Ponnighaus, J. M., Lienhardt, C., Lucas, S., Fine, P. E. \& Sterne, J. A. (1997). Comparison of bacillary indexes in slit-skin smears, skin and nerve biopsies: a study from Malawi. Int J Lepr Other Mycobact Dis 65, 211-216.
Ridley, D. S. (1955). Bacteriological interpretation of skin smears and biopsies in leprosy. Trans R Soc Trop Med Hyg 49, 449-452.

Roche, P. W., Britton, W. J., Failbus, S. S., Williams, D., Pradhan, H. M. \& Theuvenet, W. J. (1990). Operational value of serological response in multibacillay leprosy patients and bacteriological correlates of antibody response. Int J Lepr Other Mycobact Dis 58, 480-490.
Srinivas, D., Narsimha Rao, P., Laxmi, T. S. S. \& Suneetha, S. (2002). Bacterial index of granuloma and its relevance compared to BI of skin smears. Lepr Rev 73, 79-80.

WHO (1988). WHO Expert Committee on Leprosy. World Health Organ Tech Rep Ser 768, $1-51$.

WHO (1998). WHO Expert Committee on Leprosy. World Health Organ Tech Rep Ser 874, $1-43$. 